\title{
Online Interactions: Comparing Self-Disclosure and Self-Presentation between Friendship and Dating
}

\author{
Gabrielle Larson and Titus Asbury* \\ Texas Woman's University, USA \\ Submission: May 18, 2018; Published: June 08, 2018 \\ *Corresponding author: Titus Asbury, Texas Woman’s University, USA, Email: EAsbury@twu.edu
}

\begin{abstract}
Throughout the past few decades, there has been a powerful surge in technology that has had a profound impact on how people interact with one another. Due to the recent increase in technology, online interactions are reaching an all-time high. The current study examined two online behaviors: self-disclosure and self-presentation. The study assessed two types of online relationships: friendships and online romantic relationships. A total of 55 participants completed this online study through Psych Data. Participants were gathered through convenience sampling. Each participant completed an online survey that consisted of the Presentation of Online Self Scale and the Perception Control Scale. Results and implications for the type of relationships impact on online behaviors are discussed.
\end{abstract}

Keywords: Online behavior; Online interaction; Online communication; Online dating; Online relationships; Self-disclosure; Self-presentation

\section{Introduction}

Within the past few decades, there has been an enormous growth in technology around the world. The world of technology has continued to evolve and technology has become a vital piece in most individuals' lives. In most first world countries technology has become indispensable; this means life without this technology would become nearly impossible for some individuals. Technology is used for multiple purposes; work, education and pleasure all rely heavily on current technology. One major area of influence technology has had an immense impact on is its ability to connect others. It appears that the use of online communication has become integral in maintaining relationships. In order to foster online communication, websites and mobile applications have been created that allow people to meet new people as well as continue communication with people they already know. In regards to the websites and mobile applications that encourage individuals to meet new people, these sites can help people search for potential romantic partners; these sites are often referred to as online dating sites. While online dating is one way of using technology to connect with others, online communication can also be a powerful tool in connecting friends.

Websites such as Facebook, Twitter, Instagram, and Snapchat are examples of social networking sites that help link individuals together and allow personal information to be shared. Online communication encourages users to engage in both selfdisclosure and self-presentation. The prevalence of online communication in the world today leads to the question: is there a difference between self-disclosure and self-presentation among friendships and online romantic relationships?

Prior to discussing how relationship styles impact online communication, it is necessary to first define self-disclosure and self-presentation. In an article by Gibbs [1] self-disclosure is "defined as any message about the self that an individual communicates to another... individuals make themselves known to other people and, others reciprocate by sharing revealing information." Self-disclosure allows individuals to engage in intimacy with one another in an effort to further deepen their relationship [1]. Self-disclosure is a necessary aspect of relationships because it allows individuals to share information about them and connect with people on a deeper level. This type of behavior can strengthen relationships because it has the power to connect people. While self-disclosure is sharing information, self-presentation is "the processes through which individuals communicate an image of themselves to others" Yang [2]. Self-presentation also relates to self-development and selfidentity [2]. Self-presentation refers to the way an individual chooses to present themselves to others. It is vital to understand the difference between self-disclosure and self-presentation, as this current study will focus on both. Self-disclosure refers to 


\section{Psychology and Behavioral Science International Journal}

when an individual shares information with another to create intimacy, while self-presentation is how the individual chooses to portray him or her.

Self-disclosure and self-presentation are important online behaviors to assess when discussing online interactions. Varnali [3] conducted a study that further examined potential reasons individuals might engage in self-disclosure online. According to the study, self-disclosure on social networking sites has the ability to "facilitate establishing mutual understanding, intimacy, and trust in interpersonal relationships" [3]. Self-disclosure is a key factor in both romantic relationships and friendships because it allows individuals to connect on a more intimate level. Therefore, self-disclosure is a vital tool in both types of relationships because it encourages a feeling of connectedness between people. In a different study by Kim \& Lee [4], the role of self-presentation in online interactions is examined. Perhaps one of the biggest findings from the study was that self-presentation has an effect on an individual's subjective well-being. The study explained if an individual is to engage in positive and honest selfpresentation, "the psychological benefits of self-enhancement are derived" [4]. Therefore, online self-presentation can be considered a strong power because it has the ability to impact how an individual views himself or herself. Both self-disclosure and self-presentation are important factors to assess when studying online interactions because both behaviors can have an immense impact on relationships.

While not every person has engaged in online dating, the increasing popularity of online dating is apparent. The mention of online dating can be found from movies to television and even current literature. In fact, an article from 2015 states, "online dating is estimated to be a $\$ 2$ billion business, with 1 in 10 Americans reporting they have used an online dating site or mobile app" [5]. Online dating has continued to gain popularity as an effective way to find romantic relationships. Therefore, psychology has decided to take a deeper look at online dating, including both the advantages and disadvantages of participating. In regards to the relationship between online dating and selfpresentation, one of the advantages includes dating sites using a demographics questionnaire while having users create their online dating profile. These questionnaires ask about personal information in an attempt to match people based on similarities [5]. By using these demographic questionnaires at the beginning of creating online dating profiles, self-presentation is being used as a tool to match individuals together based on similar interests, hobbies, personality types, and goals. One of the major advantages of this self-presentation questionnaire is that it has the ability to make online dating easier for users since the website is able to match potential partners instead of having the users engage in extensive work to search. The study also looks at potential disadvantages of online dating; however, none of the disadvantages pertained to self-disclosure or self-presentation. Online dating is a unique source available for individuals to meet and interact with potential romantic partners; this type of online interaction has become more prevalent recently as technology has become more important in today's society.

A necessary factor in developing an online relationship is that individuals must be willing to engage in self-disclosure. An article published in 2015 discusses the importance of selfdisclosure for online relationships; the article states, "selfdisclosure becomes the only available means to communicate" [6]. The article continues to elaborate on the idea that online dating websites and mobile applications provide a comfortable environment for online interaction that allow both males and females to engage in deep levels of self-disclosure [6]. The study focuses on reasons for online self-disclosure and the factors that impact the likelihood and prevalence of it occurring. Factors such as gender, age, emotional state, and computer use all impact whether an individual will or will not engage in self-disclosure via online dating sites [6]. It is important to understand these factors because they help explain the reason why self-disclosure occurs and how it can be facilitated through online dating sites.

Online interactions encompass more than online dating relationships; in order to have a holistic view of online interactions it is necessary to also discuss friendships that engage in online interactions as well. Most of the recent research of online interactions focuses on romantic relationships, strangers, or friendships created due to the use of social media sites. This type of research is beneficial, but it leaves a gap in research because it does not address face-to-face friends who use social networking sites. A study published in 2013 supports this idea by stating, "considerable research on computer-mediated communication has examined online communication between strangers, but little is known about the emotional experience of connectedness between friends in digital environments" [7]. Although looking at stranger's online interactions is beneficial in understanding how people develop relationships without face-to-face contact, it is also necessary to look at friend relationships and examine how online interactions influence and impact their face-to-face relationships. Examining multiple types of relationships that engage in online interactions is necessary because it provides a more accurate and complete depiction of cyber psychology.

One common misconception of online interactions is the idea that strangers are the predominant users. According to a study published in 2012, adolescents use online communication with friends made through face-to-face interactions the same amount as they use this type of communication to connect with strangers [8]. One major area examined in this study was adolescents' desire and need for intimacy and emotional closeness to other individuals. The study called for participants to complete questionnaires regarding online behavior, explanation for using social networking, and how the participant believed these social networking sites impacted their friendships [8]. Based on the results from this study, it is concluded that "on average, the teens in our study interacted with $95 \%$ of their listed social 
networking site friends in face-to-face contexts, with only $5 \%$ described as people that they meet only online" [8]. The findings from this study suggest that people are likely to interact online with friends they have made from face-to-face communication. In fact, the results support the idea that people tend to interact more with people they have face-to-face interactions with rather than people they met only online. Although it is a popular thought that strangers typically engage in online interaction the most, this study supports the idea that this is merely a misconception of online interactions. This study strengthens the idea that it is necessary to look at different types of relationships when it comes to online interactions.

There are certain differences between online self-disclosure and self-presentation based on the type of relationship. An article produced by Subrahmanyam \& Greenfield [9] highlights online communication between friends; the article stated intimacy and identity play key roles in how online interactions will occur. It is important to note intimacy refers to the idea of self-disclosure and identity refers to the idea of self-presentation. While selfdisclosure is an important factor in face-to-face friendships, the article also stated that online self-disclosure impacts these types of friendships as well. The article states, " $48 \%$ of online teens believe that the Internet has improved their relationships with friends" [9]. Although this study focuses on friendships, it can be inferred that self-disclosure is also vital in online romantic relationships. A common theme in the study is the idea that selfpresentation is beneficial in creating a coherent identity and enabling self-exploration [9] Based on this information, it appears that online self-presentation is important in creating a strong emotional connection, regardless of the type of relationship. Overall, friendships with online communication seem to rely just as heavily on self-disclosure and self-presentation as online romantic relationships do.

While addressing online interactions, it becomes necessary to discuss the possibility of deception being used in online communication. The idea of deception can be considered a major limitation when it comes to online interactions because individuals' self-disclosure and self-presentation may be vastly different than their true identity. Recent research has shown that there is a strong possibility of deception being used in selfpresentation; one study highlights the likelihood of this occurring by stating, "internet communication platforms are suspected to enhance the potential of deceptive self-presentations... research has suggested that various features of online systems and the timing of messages promote distorted self-presentations by reducing immediate accountability for those distortions" [10]. The study concludes that self-presentation did in fact occur, but it also states that individual's self-presentation is not always accurate or honest. The idea of dishonesty in self-presentation is important to note because this will likely impact who people choose to engage in honest self-disclosure with. Types of relationships could potentially impact whom an individual feels comfortable enough around to provide an honest selfpresentation. One of the major reasons individuals engage in distorted self-presentations is due to social desirability [10]. Social desirability refers to the idea that individuals want to appear as socially acceptable as possible when engaging and interacting with others. Similar to self-presentation, distorted self-disclosure can also occur in online communication. The same study concludes that, "interpersonal deception has been conceptually defined as occurring when communicators control the information contained in their messages to convey a meaning that departs from the truth as they know it" [2]. It is likely this type of distorted self-disclosure can also be attributed to social desirability. Distorted self-disclosure has the ability to cause major issues in all types of relationships. Therefore, two major limitations when assessing online interactions and behaviors are dishonest self-presentation and distorted self-disclosure.

\section{The current study}

The current study is an analysis of interactions regarding likelihood of self-disclosure online and online self-presentation behaviors based on relationship type. Self-disclosure and selfpresentation will be assessed to evaluate [11] the likelihood of self-disclosure based on relationship and [10] the effect of relationship type on an individual's online self-presentation behavior. The research questions are: will an individual be more likely to engage in self-disclosure online with a friend or with a romantic partner, and does self-presentation of online behaviors differ based on relationship type?

Based on these research questions, we were able to create two hypotheses. The first hypothesis is that individuals engaging with friends will show higher levels of self-disclosure online than individuals engaging with online dating partners. The second hypothesis is that online self-perception will differ based on relationship type.

\section{Method \\ Participants}

A total of 64 participants began the online survey; however, a total of 9 participants were missing data. The 9 participants were removed from the data file and SPSS did not use the missing data; therefore, the 9 participants missing data did not impact any scores or results. A total of 55 participants fully completed the online survey, including 10 males (18.19\%) and 45 females (81.81\%). Participants were gathered through convenience sampling; the online survey was distributed via the researcher's social media sites, as well as the Texas Woman's University SONA system. There were no exclusions based on gender or ethnicity; however, participants were required to be 18 years or older. The average age of participants for males was 28.45 years old $(\mathrm{sd}=11.97)$ and for females the average age was 23.23 years old ( $\mathrm{sd}=6.58$ ). The oldest participant to complete this study was 54 years old, and the youngest participant was 18 years old. The ethnicity of participants was examined and the 
greatest numbers of participants were of the White/Caucasian/ European American ethnicity; this ethnicity had 33 participants (60\%). Hispanic/Latino(a)/Hispanic American ethnicity had the second highest number of participants with 11 (20\%). Black/ African/African American ethnicity had 6 participants (10.91\%). The lowest number of participants came from the Asian/ Asian American ethnicity, which had 3 participants (5.45\%), and the Pacific Islander/Native American ethnicity had only 2 participants (3.63\%). Out of the 55 participants, 22 individuals (40\%) stated they have engaged in online dating; however, 33 individuals $(60 \%)$ stated they have not engaged in online dating. No incentives were offered to participants for completing the survey. An electronic informed consent form was presented to participants prior to beginning the study and participants were allowed to exit the study at any time.

\section{Materials}

The online survey was available through Psych Data and consisted of three scales that attempted to measure likelihood of self-disclosure, likelihood of self-presentation, and basic demographic questions. The demographic questions were based on previous surveys and included basic information such as age, gender, and ethnicity. However, for this particular study, a question was asked whether or not a participant has engaged in online dating. This question was added to provide more information regarding the audience.

In order to provide valid and reliable questions to conduct this survey, two existing surveys were used. First, the Presentation of Online Self Scale [12] was presented to participants. This scale consisted of twenty-four questions to measure an individual's likelihood of self-disclosure in an online interaction. Participants were asked to answer the questions using a Likert Scale, which included strongly disagree, disagree, neither agree nor disagree, agree, and strongly agree.

The second measure, the Perception Control Scale [11] consisted of questions assessing an individual's opinion towards self-presentation in an online interaction. The scale consisted of twelve questions regarding how the participant believes online interactions allow them to present themselves to others. Participants were asked to answer the questions using the same Likert scale as mentioned previously for the Presentation of Online Self Scale.

\section{Procedure}

In order for the study to be conducted, the Institutional Review Board (IRB) of Texas Woman's University had to first approve the necessary documents. Once the study was approved, it was distributed through Psych Data. The participants completed the online survey through Psych Data, which combined the three questionnaires mentioned in the materials section. Since the survey was online, the participants were able to access this study at a time and place of their choosing. The survey had no time limit, but it most likely took participants approximately 15 to 30 minutes to complete. Incentives were offered to Texas Woman's University students enrolled in lower level psychology courses who inputted their TWU SONA ID; if the participants completed the study he or she was awarded 1 experimental credit for his or her participation. There were no direct benefits or incentives for participants who were not in the TWU SONA system.

When the participants first opened the survey, they were shown an informed consent form. The consent form detailed the explanation and purpose of research, included a brief description of the procedures, and discussed potential risks and benefits. Finally, the informed consent offered the researchers email so if any participants had any questions or concerns regarding the study they were able to contact the researcher. After reading the information above, the participant was to indicate their willingness to complete the study. If an individual agreed to participate, they were offered a space to enter their Texas Woman's University SONA ID so they would receive credit. However, if the individual was not participating in the study for research credit in SONA, they refrained from answering said question and continued to the first part of the survey. Participants were allowed to stop the survey at any point for any reason. If a participant was not willing to complete the survey, they were advised to exit the survey before continuing any further.

Following the informed consent, participants were presented with the three scales mentioned in the materials section. The first questionnaire addressed basic demographic questions including age, gender, ethnicity, and whether or not the participant has or has not participated in online dating. After completing this portion of the study, the participant was randomly directed to one of three prompts regarding how the participant feels about himself or herself in an online world. The three prompts asked participants to answer the next survey items based on the prompt presented. Prompt 1 asked about online interactions with friends, prompt 2 asked about online interactions with romantic partners, and prompt 3 asked about general online interactions. The friend and romantic prompts were considered the experimental groups and the final prompt about general online interactions was the control group. Participants were then required to answer the Presentation of Online Self Scale [12] this survey consisted of twenty-four questions meant to assess an individual's likelihood of self-disclosure in an online interaction.

The last questionnaire attempted to find information about online self-presentation. The participant was presented with instructions to answer the following questions with the most accurate description of them in an online world. This portion of the survey consisted of twelve questions meant to assess online self-presentation. After completely answering the survey, participants were debriefed on information about the study and again, were given contact information for the researcher. 


\section{Psychology and Behavioral Science International Journal}

Once over fifty participants completed the survey, the data was analyzed.

\section{Results}

(Figure 1-4) both hypotheses were tested using a one-way ANOVA analysis to help determine if there were any significant results. Prior to performing the ANOVA, the study required the researcher to compute two variables. Adding together the twenty-four questions asked on the Presentation of Online Self Scale created the "POSS Total" and adding together the twelve questions from the Perception Control Scale created the "PCS Total."

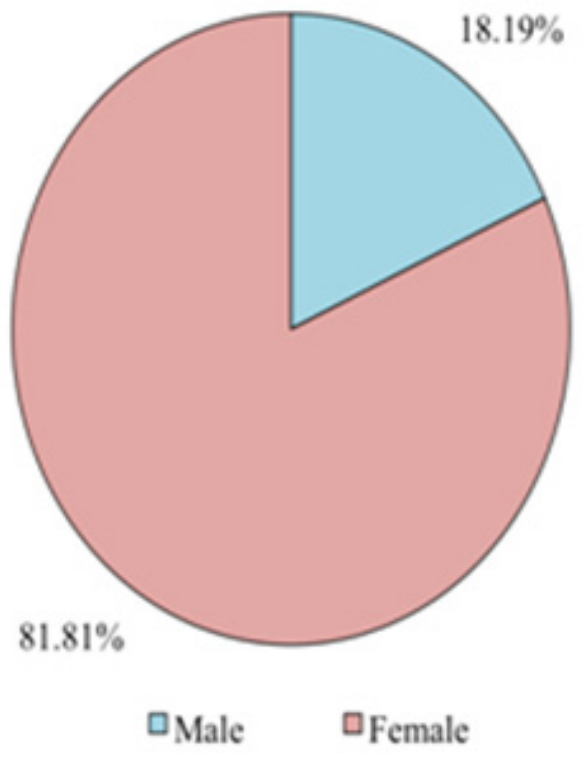

Figure 1: Demographics gender by percent.

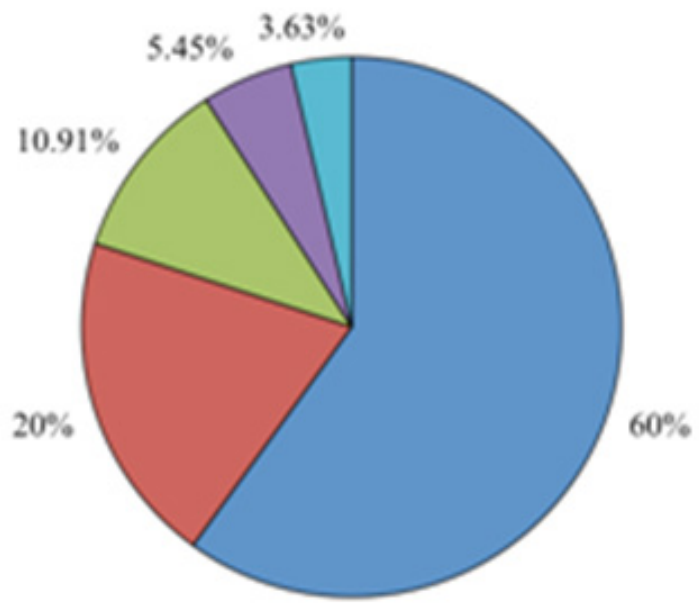

White/Caucasian/European

ם Hispanic/Latino/Latina/Hispanic American

口 Black/African/African American

- Asian/Asian American

QPacific Islander/Native American

Figure 2: Demographics race/ ethnicity by percent. 


\section{Psychology and Behavioral Science International Journal}

\begin{tabular}{|c|c|c|c|c|c|}
\hline $\begin{array}{c}\text { Dependent } \\
\text { Variable }\end{array}$ & $\begin{array}{c}\text { Random } \\
\text { Stimulus I }\end{array}$ & $\begin{array}{c}\text { Random } \\
\text { Stimulus II }\end{array}$ & $\begin{array}{c}\text { Mean Difference } \\
\text { (I-II) }\end{array}$ & Standard Error & Significance \\
\hline \multirow{6}{*}{$\begin{array}{l}\text { Presentation of } \\
\text { Online Self Scale }\end{array}$} & \multirow[t]{2}{*}{ Friends } & Romantic & 1.81513 & 2.63 & .770 \\
\hline & & General & $-7.41176^{*}$ & 2.76 & .026 \\
\hline & \multirow[t]{2}{*}{ Romantic } & Friends & 1.81513 & 2.63 & .770 \\
\hline & & General & -5.59664 & 2.63 & .093 \\
\hline & \multirow[t]{2}{*}{ General } & Friends & $7.41176^{*}$ & 2.76 & .026 \\
\hline & & Romantic & 5.59664 & 2.63 & .093 \\
\hline \multirow{6}{*}{$\begin{array}{l}\text { Perception } \\
\text { Control Scale }\end{array}$} & \multirow[t]{2}{*}{ Friends } & Romantic & -.32440 & 2.29 & .989 \\
\hline & & General & -4.44485 & 2.40 & .164 \\
\hline & \multirow[t]{2}{*}{ Romantic } & Friends & .32440 & 2.29 & .989 \\
\hline & & General & -4.12045 & 2.25 & .170 \\
\hline & \multirow[t]{2}{*}{ General } & Friends & 4.44485 & 2.40 & .164 \\
\hline & & Romantic & 4.12045 & 2.25 & .170 \\
\hline
\end{tabular}

*. The mean difference is significant at the 0.05 levels.

Figure 3: One way ANOVA results.

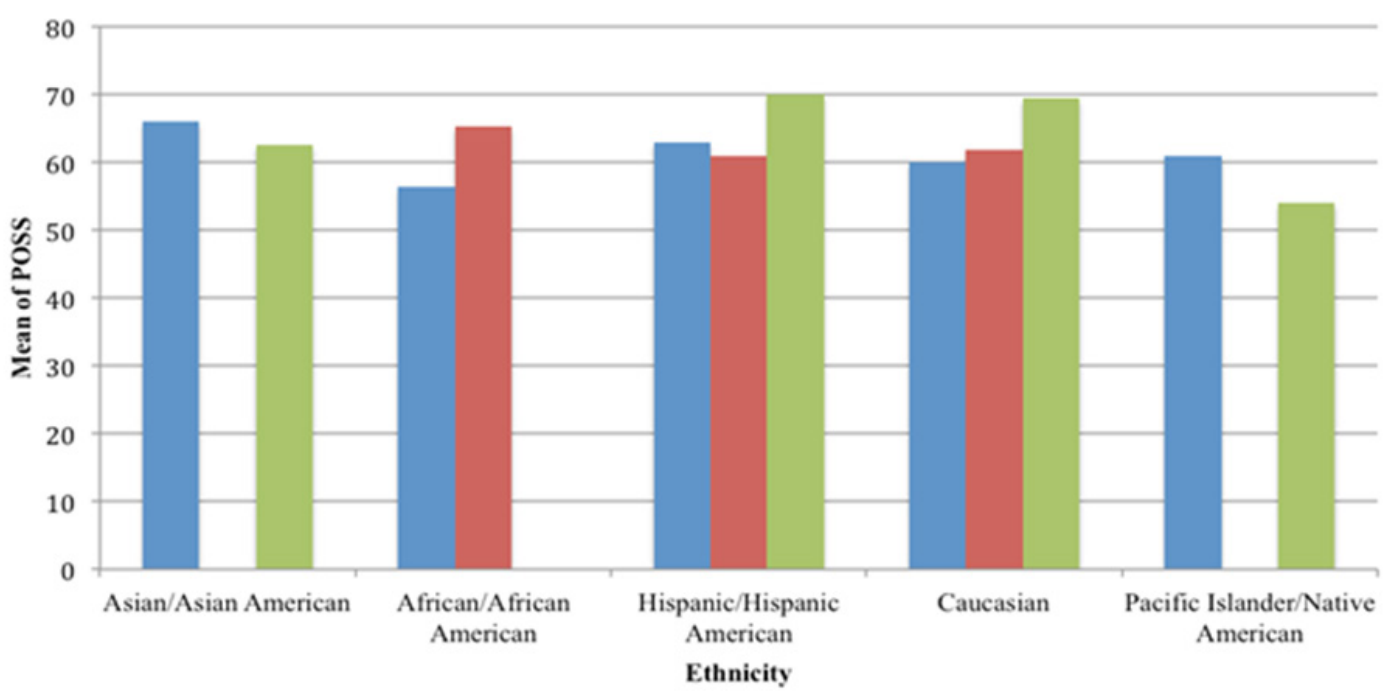

m Friends $=$ Romantic $=$ General

Figure 4: POSS scores by ethnicity.

In regards to the first hypothesis, a one-way between subjects ANOVA was conducted to compare the effects of relationships type on self-disclosure. There was a significant effect of relationship type on self-disclosure online at the $\mathrm{p}<.05$ level $[F(2,52)=3.960, p=.025]$. Post hoc comparisons using the Tukey HSD test indicated that the mean score for the friendship condition $(M=60.47, \quad S D=2.76)$ was significantly different than the general condition $(M=67.88, S D=2.76)$. However, the romantic condition $(\mathrm{M}=67.88, \mathrm{SD}=2.63)$ did not significantly differ from the friendship or general conditions. Therefore, the hypothesis was not supported; the results did show a significant difference between general online interactions and friend interactions in comparison to general online interactions and romantic interactions. A one-way ANOVA was also performed to assess Perception Control Scale. A one-way between subjects ANOVA was conducted to compare the effect of relationship type on self-presentation in online interactions. There was not a significant effect of relationship type on elf-presentation in online interactions for all of the conditions $[F(2,52)=2.231, p=.118]$. Therefore, the second hypothesis was not supported based on these results obtained from the one-way ANOVA.

Other analyses were conducted to delve deeper into other information that could be potentially beneficial for future research using this pilot study. Several t-tests were performed to analyze data and attempt to find statistical significance in other areas beyond what was looked at for the hypotheses. An independent-samples t-test was conducted to compare age and gender. There was not a significant difference in scores for 
males $(M=28.45, S D=11.97)$ and females $(M=23.23, S D=6.58)$; $\mathrm{t}(61)=2.036, \mathrm{p}=.046$. An independent-samples t-test was conducted to compare Presentation of Online Self Scale scores and gender. There was not a significant difference in scores for males $(M=60.30, S D=7.18)$ and females $(M=64.16, S D=8.66)$ completing the POSS; $\mathrm{t}(54)=-1.309, \mathrm{p}=.196$. An independentsamples t-test was also conducted to compare Perception Control Scale scores and gender. There was not a significant difference in scores for males $(M=40.30, S D=8.27)$ and females $(\mathrm{M}=42.34, \mathrm{SD}=6.80)$ completing the PCS; $\mathrm{t}(54)=-.823, \mathrm{p}=.414$. Based on these analyses there was no additional information to be assessed.

However, one area of interest after running data was the results from a Univariate general linear model between ethnicity and the POSS. While the descriptive statistics did not show any significance, there were some interesting conclusions that can be drawn from these analyses. It is important to note that the small number of participants affects these conclusions; therefore, a larger study would need to be conducted to see if these results can be considered reliable and valid. The Univariate general linear model was completed between ethnicity and POSS. The results showed Asian/Asian American participants are most likely to engage in self-disclosure in general online interactions $(M=63.67, S D=3.21)$. African American participants are most likely to engage in self-disclosure in friendship interactions $(M=60.83, S D=12.51)$. Hispanic/Hispanic American participants are most likely to self-disclose in general interactions $(M=65.81$, $\mathrm{SD}=7.81$ ). Caucasian participants are also more likely to engage in self-disclosure in general online interactions $(M=63.48$, $\mathrm{SD}=8.43$ ). Finally, Native American/Pacific Islander participants are slightly more inclined to engage in self-disclosure with friends $(M=57.50, S D=4.95)$. However, the small number of participants greatly impacts the results from this analysis.

\section{Discussion}

Assessing the data helped create conclusions based on our hypotheses. The first hypothesis was that friends would show higher levels of self-disclosure online than individuals engaging with online dating partners; however, this hypothesis was not supported by the results. Our hypothesis was based on a similar study by Reich et al. [8] that reported the use of self-disclosure online between friends was vital and occurred more often than between strangers. However, we failed to find the same differences between online interactions.

The second hypothesis was that online self-presentation would differ based on relationship type. Our hypothesis was based on an earlier study by Subrahmanyam \& Greenfield [9], which suggested online self-presentation was important regardless of relationship type. While we wanted to determine if online self-presentation varied across relationship type, we did not find differences between friends, romantic interests, and general acquaintances.
Therefore, the study by Subrahmanyam \& Greenfield [9] actually did support our findings that relationship type did not impact online self-presentation.

\section{Limitations}

While conducting any study, it is inevitable that there will be certain limitations that should be addressed. This study is no exception, and therefore, it is necessary that limitations are discussed and how these limitations might have possibly impacted the results. One major limitation is the fact that this was a pilot study. This means that the number of participants was somewhat low and therefore, this study can be considered a preliminary study that can help future researchers become aware of the feasibility of this study. The small sample size made it difficult to find significant relationships from the data.

A second major limitation is that this study is self-report. The survey presented to the participants asked for individuals to provide information regarding themselves and their online habits; however, self-report surveys can cause issues if a participant does not answer honestly or is unable to provide accurate information for the questions. The study also faced lack of data, since 10 participants had missing data and were not analyzed. This could be attributed to the fact that the study could have caused experimental fatigue. This means either the length, depth of questions, or format of the study could have caused mental fatigue. This mental fatigue could be a reason for participants not fully completing the survey to its entirety. Along with the fact that this study is using a self-report measure, participants might also engage in social desirability bias. This type of bias could mean an individual might respond based on whether or not an answer will make them appear favorably to others. Although it was stated this study would be anonymous, some individuals still feel the need to respond in a positive light. Therefore, the study being self-report could have led to social desirability bias in participants.

A third limitation of this study was the disproportion between gender and ethnicities. After reviewing the results, it is apparent that more females participated in this study than males. Since this study was available on Texas Woman's University SONA site, it is likely that the reason more females participated in this survey is because more females attend this university. In regards to ethnicity, the results also showed high amounts of both Caucasian and Hispanic ethnicities, but low amounts of African American, Asian, and Native American. A goal of all studies is for the results and conclusions to apply to the greater population. In order for a study to be applied to a bigger population, it is important to have a relatively even number of ethnicities. Therefore, the disproportion of gender and ethnicities impacted how the results can be applied.

Finally, a fourth limitation stems from the measurements and instructions. It is possible that participants interpreted statements and questions in different ways; therefore, causing 
participants to answer in ways not desired by the researcher. However, it is difficult to tell if participants were able to understand the questions and instructions since answers were shown on a Likert scale. The likelihood that participants misinterpreted questions and instructions can impact the validity and reliability of this study.

\section{Future directions}

In order to increase the validity of this study, it is necessary to produce further studies. Future studies are important because they have the ability to change the mistakes in the pilot study and possibly apply the findings to a larger population. For this particular study, future researchers should look at three things: expanding the study, the relationship between online dating and online behaviors, and the relationship between sexual orientation and online behaviors. If future researchers could incorporate some of these aspects into their future studies, it would provide more information on the topic of online interactions.

As mentioned previously, it is vital that studies use a sample that is representative of all types of individuals. A study is difficult to apply to bigger populations when it is comprised of a small sample. Therefore, it would be necessary that every study that uses this pilot study include an equal representation of individuals based on age, gender, and ethnicity. The more diverse and more number of participants in the study, the easier it will be to apply the findings to a bigger population.

A second suggestion for future research includes a question asked in the pilot study; however, it would be interesting to have the topic looked at in more depth. While the focus of the pilot study was focused on assessing types of relationships and how that impacted online behaviors, it would be fascinating to see how online dating impacted individuals' online behaviors. This future study could assess whether online dating increases, decreases, or has no effect on the likelihood of self-disclosure and self-presentation occurring in online interactions. It might be helpful to focus solely on individuals who have engaged in online dating; preferably having diverse participants who have used online dating for different lengths of time. The survey might include demographic questions, the POSS, the PCS, a question regarding length of online dating, and asking the participant to use a Likert scale to express their experience with online dating. Overall, this future study would be important for cyber psychology because it would help explain the impact of online dating on future online behaviors.

Finally, examining sexual orientation's possible relationship to online behaviors would be useful as a future research study. As there has been an influx in technology, it also appears that there is an influx in research studies assessing sexual orientation's effects on an individual's life. It would be interesting to research further in depth if sexual orientation impacts online behaviors. This type of research study could include questionnaires such as demographics, the POSS, the PCS, sexual orientation, and whether or not the individual's sexual orientation is honestly presented in everyday life (i.e. do friends and family know your true sexual orientation?). Overall, this type of research study could be beneficial in assessing whether or not sexual orientation has a relationship to online behaviors; therefore, this study could examine two prevalent topics in today's society.

\section{Conclusion}

As a result of this study, we were able to take a deeper look into the field of cyber psychology. Our first hypothesis was not supported; however, one piece of data was statistically significant. This showed that people were more likely to engage in self-disclosure in general online interactions rather than in friendship interactions. Friendships and online romantic partners did not show any significant difference in engaging in online self-disclosure. The second hypothesis was not supported and none of the data was statistically significant. There was no significant effect of online self-perception based on relationship type. This study should be reproduced with a larger and more diverse population in order to ensure reliability and validity with these results.

\section{References}

1. Gibbs JL, Ellison NB, Heino RD (2006) Self-presentation in online personals: the role of anticipated future interaction, self-disclosure, and perceived success in internet dating. Communication Research 33(2): 152-177.

2. Yang CC, Bradford BB (2016) Online self-presentation on facebook and self development during the college transition. J Youth Adolesc 45(2): 402-416.

3. Varnali K, Toker A (2015) Self-disclosure on social networking sites. Social Behavior \& Personality: An International Journal 43(1): 1-13.

4. Kim J, Lee J (2011) The facebook paths to happiness: effects of the number of facebook friends and self-presentation on subjective wellbeing. Cyberpsychology, Behavior and Social Networking 14(6): 359364.

5. Wiederhold BK (2015) Twenty years of online dating: current psychology and future prospects. Cyberpsychology, Behavior \& Social Networking 18(12): 695-696.

6. Desjarlais M, Gilmour J, Sinclair J, Howell KB, West A (2015) Predictors and social consequences of online interactive self-disclosure: a literature review from 2002 to 2014. Cyberpsychol Behav \& Soc Netw 18(12): 718-725.

7. Sherman LE, Michikyan M, Greenfield PM (2013) The effects of text, audio, video, and in-person communication on bonding between friends. Cyberpsychology 7(2): 1-12.

8. Reich SM, Subrahmanyam K, Espinoza G (2012) Friending, iming, and hanging out face-to-face: overlap in adolescents' online and offline social networks. Dev Psychol 48(2): 356-368.

9. Subrahmanyam K, Greenfield P (2008) Online communication and adolescent relationships. Future Child 18(1): 119-146.

10. Andrea DC, Tom TS, Liang YJ, Levine TR, Walther JB (2012) When do people misrepresent themselves to others? the effects of social desirability, ground truth, and accountability on deceptive selfpresentations. Journal of Communication 62(3): 400-417.

11. Attrill W, Keep (2006) The perception control scale.

12. Fullwood C, James B, Chen WJ (2016) Self-concept clarity and online self-presentation in adolescents. Cyberpsychol Behav Soc Netw 19(12): 716-720. 
(C) This work is licensed under Creative BY DOI: 10.19080/PBSIJ.2018.09.555761
Your next submission with Juniper Publishers will reach you the below assets

- Quality Editorial service

- Swift Peer Review

- Reprints availability

- E-prints Service

- Manuscript Podcast for convenient understanding

- Global attainment for your research

- Manuscript accessibility in different formats ( Pdf, E-pub, Full Text, Audio)

- Unceasing customer service

Track the below URL for one-step submission https://juniperpublishers.com/online-submission.php 